\title{
experiencias sobre automación de hornos rotatorios con intercambiadores de calor en suspensión gaseosa
}

(Continuación)

W. GORTZ, Colonia

\section{DISTRIBUCION AUTOMATICA DEL CRUDO}

Los problemas que se presentan en la dosificación automática de la proporción de polvo crudo son diferentes para cada material. En primer lugar se puede aplicar el principio de que la dosificación de materias primas ha de comenzar en la trituración.

Los análisis de los sondeos y voladuras determinan la calidad de los materiales de los yacimientos con ayuda de un sistema de cubicación adecuado. Después de la extracción y de la trituración previa del material debe decidirse, según su composición química, si la materia prima se ha de almacenar separadamente o se ha de mezclar proporcionalmente en montones ${ }^{12}$. La experiencia ha demostrado que, en la mayor parte de los casos, la solución más ventajosa es un almacenamiento separado y la toma posterior de cantidades calculadas de las diversas materias.

Por este medio únicamente es posible, sin embargo, conseguir una aproximación en la composición del crudo deseado.

Las materias primas que se almacenan en los depósitos nunca se conocen con exactitud en cuanto a su calidad. Esta se ha de determinar periódicamente por análisis de laboratorio. Después de la molienda de la materia prima, se efectúa la determinación del contenido de cal o un análisis completo, pero no con gran frecuencia.

Según los resultados conseguidos, se realiza la homogeneización. Sin embargo, se ha comprobado que este método no basta para mantener constante el nivel de cal o los diversos módulos ${ }^{13}$. La instalación del horno disminuye, generalmente, las desviaciones que aún existen; no obstante, a veces, aparecen los siguientes fenómenos:

1) marcha irregular del horno;

2) formación de costras;

3) obstrucción del intercambiador de calor. 
Estos inconvenientes se reducen si una distribución automática del polvo crudo mantiene constante la proporción de cal y de los restantes componentes del clínker. Como ejemplo puede servir la figura 9, en la cual se exponen las instalaciones experimentales con una instalación de intercambio de calor. La forma de la distribución automática anterior y posterior de las materias primas puede deducirse de dicha figura.

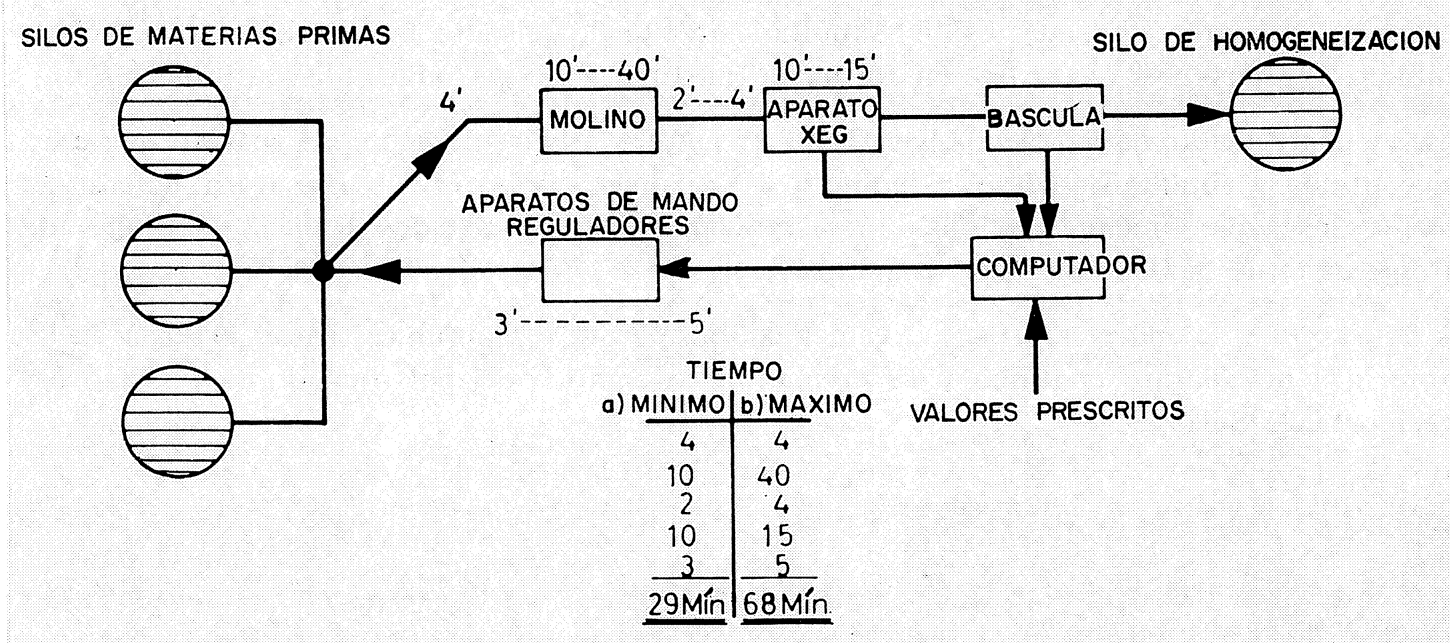

Fig. 9.-Circuito de regulación de la distribución proporcional del polvo crudo.

\section{REALIZACION DE LA DISTRIBUCION AUTOMATICA PROPORCIONAL DEL CRUDO}

Para un control eficaz de la calidad del crudo, que constituye la base para una distribución automática proporcional del mismo, se requiere el análisis por fluorescencia de rayos $\mathrm{X}^{14}$. El fundamento de este método está representado en la figura 12.

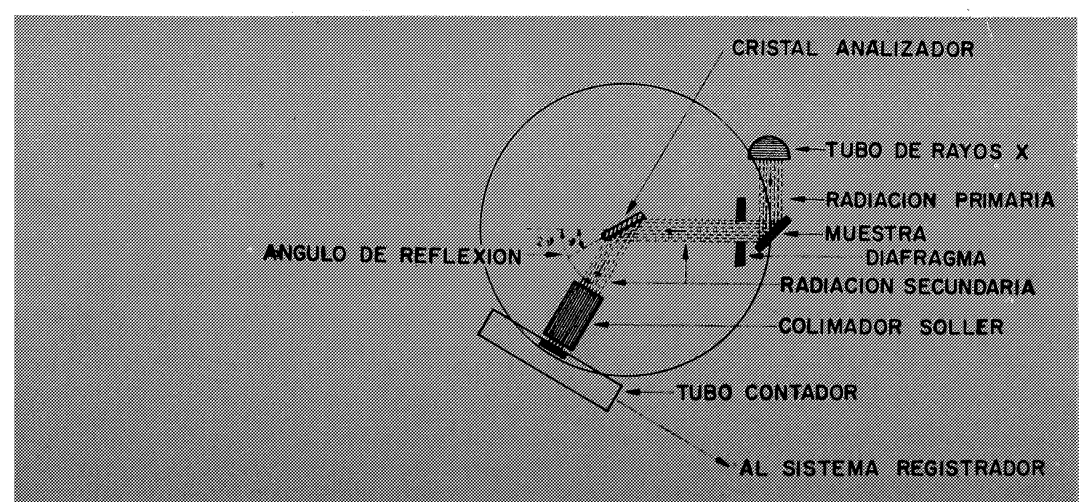

Fig. 12.-Fundamento del análisis por fluorescencia de rayos $X$

De la muestra irradiada con los rayos $\mathrm{X}$ surge una radiación secundaria. La frecuencia de esta radiación en el espectro se dispone según los elementos existentes y su situación en el sistema periódico. Mediante la reflexión de la radiación en un cristal analizador se separa una determinada longitud de onda del espectro de dicha radiación. El ángulo de ajuste del cristal es un índice para conocer el elemento, y la intensidad de la radiación representa una medida de la cantidad de dicho elemento. 
Hasta ahora se han difundido mucho los aparatos de funcionamiento discontinuo, con los que pueden medirse todos los elementos o sus combinaciones por medio de un desplazamiento del cristal analizador; dichos elementos se han de encontrar en el sistema periódico desde el número 13 hacia adelante. La muestra se prensa en forma de pastilla y se coloca, manual o) automáticamente, en el aparato.

Un tipo distinto de estos aparatos posee varios cristales (por ejemplo, de 6 a 8 ), que sin desplazar el aparato pueden medir simultáneamente de seis a ocho elementos. La probeta también se prensa, en este caso, en forma de pastilla y se introduce discontinuamente.

Para el control automático del polvo crudo sə emplea preferentemente un aparato que efectúa, continua y simultáneamente, el número de análisis necesarios. Un aparato acreditado de esta clase, que funciona en unas 15 instalaciones de los Estados Unidos y en una de Europa, es el de la General Electric Company.

Las figuras 10 y 11 muestran, respectivamente, un aparato discontinuo para el análisis de laboratorio y un aparato continuo para el mando automático del polvo crudo en una fábrica de cemento de Suiza.

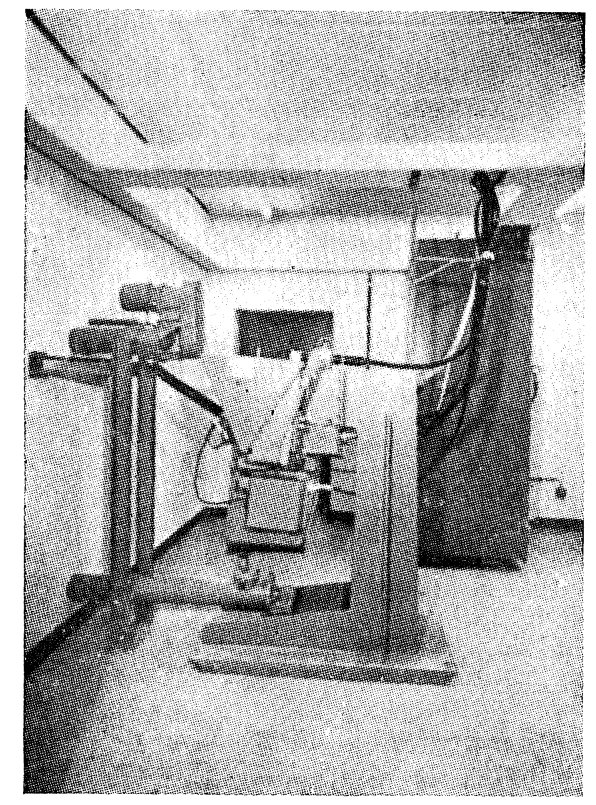

Fig. 10.-Aparato continuo de fluorescencia de rayos $X$.

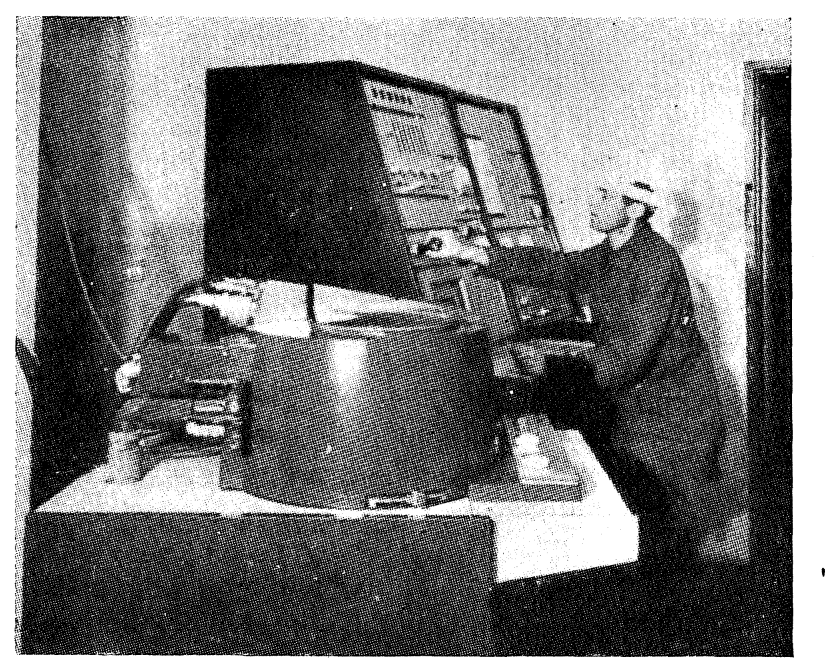

Fig. 11.-Aparato discontinuo de fluorescencia de rayos $X$ para trabajo de laboratorio.

Cuando no se presentan proporciones un poco especiales, puede hacerse el análisis permarente de polvo crudo de cemento con la sigu iente exactitud:

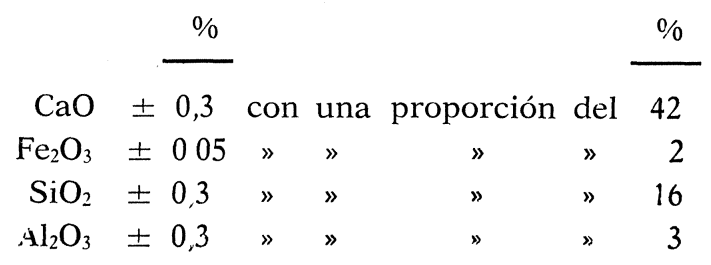


En el análisis por fluorescencia de rayos $\mathrm{X}$ aparecen mayores inexactitudes cuando un determinado elemento o su combinación se presenta en varias fases y su composición varía con el tiempo. Sobre todo estas dificultades aparecen con el silicio, que unas veces está en forma de cuarzo y otras en forma de minerales arcillosos. De esta manera pueden obtenerse errores hasta de varias unidades por ciento. Para dominar técnicamente esta situación, se determina la proporción variable de cuarzo haciendo un a nálisis por difracción de rayos $\mathrm{X}$ que se utiliza como patrón para la corrección del valor del siiiicio obtenido erróneamente.

En la figurc. 13 se puede ver el fundamento de la medición en el análisis por difracción de rayos $\mathrm{X}$.

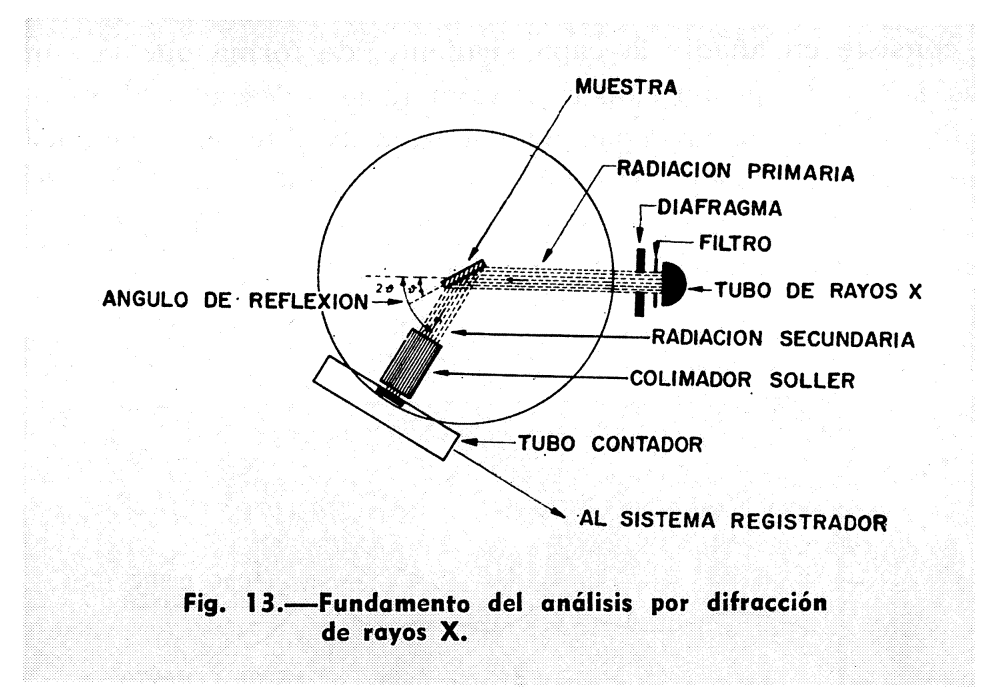

Al contrario que en el análisi. por fluorescencia de rayos $\mathrm{X}$, en el análisis por difracción de rayos $\mathrm{X}$ se determina la composición de las fases mediante la fijación de las distancias entre ios planos de la red (Valores-d) ${ }^{13}$.

Una medida permanente y automática de la calidad del crudo presenta el problema de una toma de muestra adecuada de la materia prima. Fundamentalmente se consideran dos puntos de toma: delante y detrás del molino ${ }^{14}$. Como la afluencia automática de la mezcla de materias primas debe realizarse, en cada caso, delante del molino, y precisamente en los puntos de toma de los depósitos, parece más razonable, en primer lugar, efectuar también la toma de muestra delante del molino; de esta forma, el tiempo de permanencia del material en el molino no obstaculiza el desarrollo de la regulación. Sin embargo, en este caso se presentan las siguientes dificultades:

1) como el tamaño de grano del material delante del molino se encuentra entre 0 y 25 ó $40 \mathrm{~mm}$, ha de retirarse una gran cantidad de muestra para que ésta sea representativa;

2) la muestra se ha de secar, moler y cuartear para el análisis automático.

En la toma de muestra detrás del molino desaparecen estas dificultades. Con tomamuestras adecuados puede retirarse una cantidad de polvo crudo representativo de unos $100 \mathrm{~kg} / \mathrm{h}$. La finura del polvo crudo basta para el aparato analizador. Una desventaja es que debe considerarse el gran tiempo de permanencia ya citado del material en el molino. No obstante, este inconveniente puede resolverse técnicamente. 
En la figura 9 se muestra el esquema de regulación para la distribución automática proporcional del crudo.

En dicha figura el lugar de toma de muestra se encuentra detrás del molino. Los tiempos de permanencia indicados para cada fase del proceso muestran, claramente, que no puede conseguirse una regulación continua de la calidad del crudo, proporcionando la extracción de los depósitos delante del molino por los medios tradicionales de la técnica de regulación. Por esta razón, los datos sobre la calidad del polvo crudo van en primer término a un computador. Este recibe simultáneamente la medida de las cantidades de materias primas que están dispuestas para una determinada calidad del material. El computador también recibe información de las diferentes capas de material que entran en el silo de homogeneización. Su misión consiste en añadir la capa siguiente, de forma que la suma de las composiciones de todas las capas proporcionen el valor teórico deseado. Al final de la operación de llenado en el silo se encuentran capas de material de diferente composición, que después de la homogeneización proporcionan una mezcla de polvo con la calidad deseada; con una regulación correcta puede alcanzarse el valor teórico con una tolerancia del $\pm 0,5 \%$, referida al carbonato cálcico calculado (figura 14).

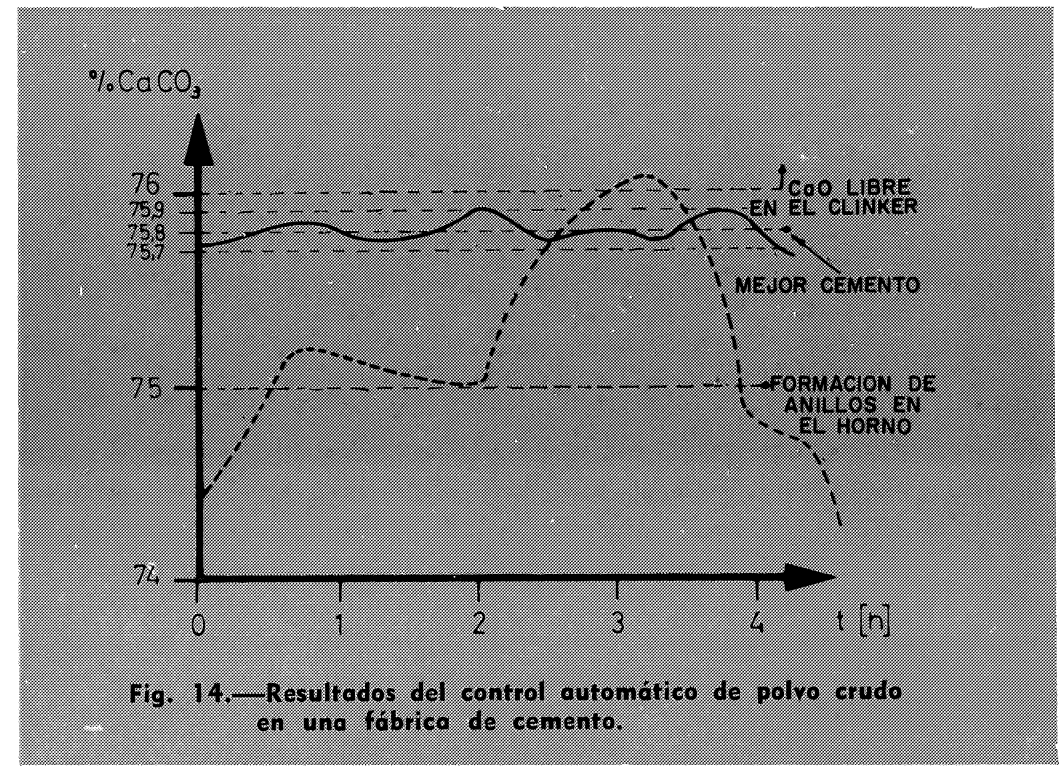

Para el cálculo es necesario un computador relativamente más pequeño, que trabaja según el principio digital de cálculo. Una instalación, que está provista de dispositivos de regulación y control de tipo convencional y de distribución automática proporcional de polvo crudo, puede considerarse como preferente. Un control amplio del computador, que se extiende al horno, al intercambiador de calor y al enfriador, puede crear más inestabilidad a la instalación debido a cambios en la cantidad de combustible y en el número de revoluciones. Las oscilaciones de las materias primas no pueden compensarse cambiando las condiciones de transformación de las materias primas en el horno rotatorio, de forma que resulte un clínker de propiedades constantes. Como único método puede emplearse el de la compensación de las oscilaciones de materias primas en la sección de preparación de crudos. 


\section{SITUACION Y MONTAJE DE UNA CENTRAL DE CONTROL}

En la agrupación de todas las instalaciones de mando y regulación del intercambiador de calor en suspensión gaseosa, del molino y de los dispositivos auxiliares, se presenta, en primer lugar, el problema de localizar el lugar correcto para la central de control.

Si una instalación de cocción se rige por una central de control, el lugar correcto está sin duda en la plataforma del hornero. Para agrupar varias instalaciones de hornos en una sala de control es necesario, en general, colocar un edificio central entre los hornos.

La disposición de la sala de mando debe realizarse de manera que la persona que manipula la producción pueda observar, desde un punto, toda la instrumentación y las imágenes luminosas. Las salas alargadas y en forma de tubo son inadecuadas. En el caso de paneles de conexiones alargados es más razonable la disposición en U o en semicírculo

En las figuras 15 y 16 se han reproducido dos salas típicas de control.

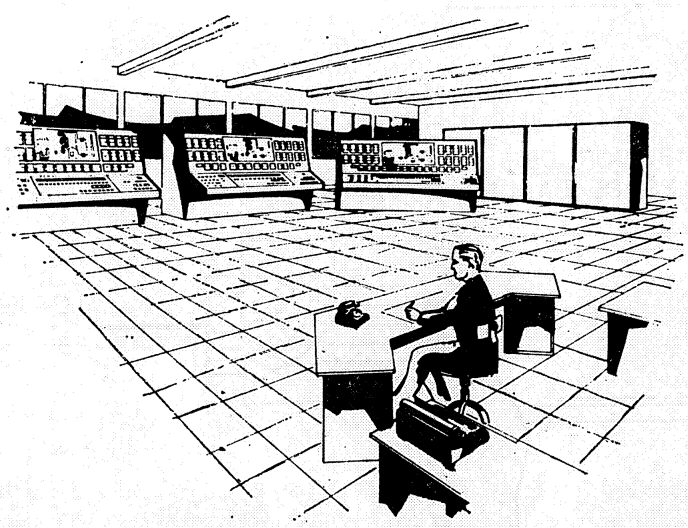

Fig. 15. - Sala de control con pupitres de mando largos.

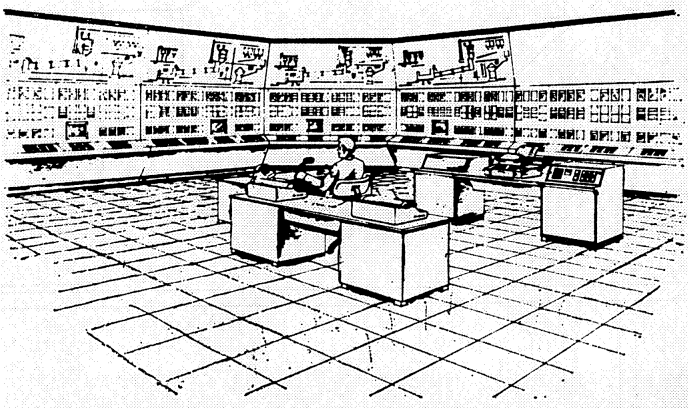

Fig. 16.-Sala de control en forma de montaje compacto.

En la figura 15 todo el esquema de producción está dispuesto en una pantalla luminosa. La representación empieza por la cinta de alimentación que proviene de la trituración en cantera y acaba delante del ensacado. La instalación tiene tres hornos, cuatro molinos de crudo y cuatro molinos de cemento. Para no interrumpir ni prolongar innecesariamente el curso de la imagen mediante la instrumentación, tocios los instrumentos están dispuestos en una pared situada en frente. Este montaje es fácil de supervisar. Sin embargo, presupone una amplia planificación de la sala.

En la figura 16 se ha efectuado en las paredes una disposición muy densa. El montaje se ha llevado a cabo en tres filas: la fila superior muestra la instalación por medio de un esquema luminoso; la intermedia, la instrumentación; y el tablero de mando inferior, los interruptores correspondientes. Esta disposición tiene, además de la ventaja de necesitar poco espacio, la facultad de una fácil observación de las imágenes luminosas, de los instrumentos y de los interruptores.

La sala central de control de instalaciones mayores ha de tener un piso de cables. Los cables que llegan allí se conducen a un distribuidor de señales. Este método de instalación 
puede observarse en la figura 17. El distribuidor de señales tiene como misión unir debiaamente los conductores que llegan y salen, y de fijar los cables aislados en orden numérico sin considerar su destino. El personal de entretenimiento puede comprobar cada conexión de cables y cada tensión en el piso de cables, sin entrar en la sala de control.

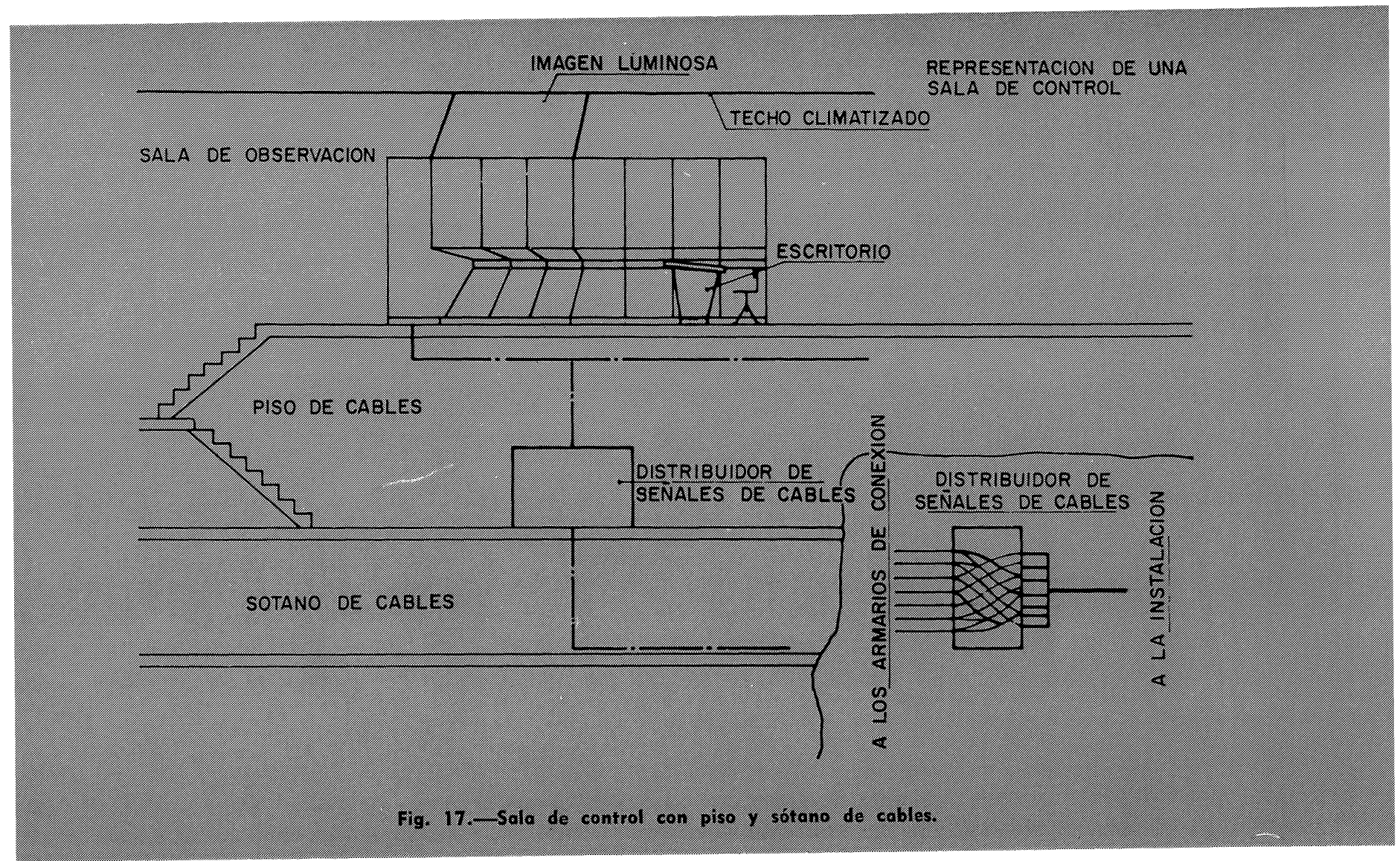

\section{CONCLUSION}

Las posibilidades señaladas para la automación de hornos con intercambiadores de calor en suspensión gaseosa proporcionan un ahorro de personal y un aumento de la producción. En los Estados Unidos se utilizan de esta forma solamente 0,4 horas de trabajo por tonelada de cemento. Una instalación montada en Suiza permite obtener en 0,6 horas de trabajo 1 tonelada de cemento. Los aumentos de producción se consiguen, en primer lugar, mediante la reducción de los tiempos de parada en el caso de emplear una materia prima irregular. Además, la instalación siempre puede ponerse en los límites superiores de rendimiento cuando los inconvenientes por parte de las materias primas son eliminados.

La automación de una instalación requiere una estrecha colaboración entre el constructor y el director de fabricación. El montaje sólo puede realizarse en etapas sucesivas bien definidas. Antes de esto debe advertirse la posibilidad de solucionar este problema a tanto alzado. En la construcción de nuevas instalaciones debería preverse en el proyecto todo lo referente a una posible automación posterior. 
El desarrollo de la industria del cemento se dirige indudablemente a la construcción de grandes unidades de producción y a una amplia automación; sin embargo, no se pueden reemplazar por ningún dispositivo automático los vigilantes de la sala de conexiones, el persorial que atiende al intercambiador, los electricistas, los engrasadores y algún otro personal auxiliar.

El mando por un solo botón desde el dormitorio del propietario de la fábrica es pura utopía.

\section{DISCUSION A ESTA EXPOSICION DE U. HAESE}

Respecto al problema de la precisión de los a paratos continuos por fluorescencia de rayos X, se han expresado dudas en diversos lugares sobre su empleo masivo en el análisis de crudo.

A menudo aparecen efectos de la matriz, que tampoco pueden corregirse por el examen de fases empleado hasta ahora. Entonces, pues, solamente se puede efectuar el análisis por fluorescencia en buenas condiciones después de la transformación de las fases por medio de la vitrificación. A pesar de esto, debería controlarse siempre la exactitud del aparato por contraste con otros métodos.

El ponente replicó que en un examen que abarcaba alrededor de 200 muestras de polvo crudo solamente aparecieron del 10 al $15 \%$ de efectos de la matriz de tal magnitud, que no podían definirse sin una corrección basada en los análisis de difracción o en la transformación de las fases de los componentes de las materias primas. De este resultado se deduce, que la frecuencia de las materias primas que presentan más facultades no es tan grande como para que no pueda hablarse de utilización amplia.

Además de esto puede aumentarse el dominio sobre los efectos de la matriz mediante el empleo de aparatos adicionales.

$\mathrm{La}$ exactitud del $\pm 0,3 \%$ en $\mathrm{CaO}$ se basa en un supuesto error del $\pm 0,1$ al $0,15 \%$ en $\mathrm{CaO}$ $\epsilon n$ el análisis por vía húmeda. Dicha exactitud no ha de cambiarse con la capacidad reproductiva del resultado del aparato, que se encuentra en $\pm 0,1 \%$ en $\mathrm{CaO}$ y es un dato para la operación de regulación.

\section{RESUMEN}

La automatización de los hornos con intercambiadores de calor en suspensión gaseosa supone, como hipótesis, que el equipo de regulación y medida de los diferentes sectores de İa instalación se elija racionalmente y funcione con seguridad. Por lo tanto, en primer lugar se describen los equipos de regulación y medida más convenientes según la experiencia obtenida hasta ahora.

Como un sistema de intercambio de calor no tiene ninguna posibilidad mecánica de intervención que pueda ejercer una influencia sobre el material durante el tiempo de paso, es especialmente importante alimentar el intercambiador de calor con una materia prima de calidad constante; por ello, la segunda parte trata de las posibilidades de homogeneización automática de polvo crudo e informa sobre las experiencias llevadas a cabo con esta automación.

En la últıma parte se estudia la utilización y el equipo más conveniente para las salas de control de las fábricas de cemento. Por otra parte se hace referencia a las experiencias realizadas hasta hoy para aumentar la producción y reducir el personal, que han de lograrse con las salas centrales de control. 


\section{bibliografía}

1 Voos, E., und Grosser, F.: Eine neuartige Dosiermaschine. Zement-Kalk-Gips 8 (1955), 268.

2 Merkblatt des VDZ, MT 4: Fliesdosiereinrichtungen, 1957.

3 Voos, E.: Betrieb von Mahlanlagen mit Inertgas. Zement-Kalk-Gips 17 (1964), 526.

4 Merkblatt des VDZ, Messtechnik 02.3, 1958.

5 I.iesegang, W., und Lusche, K.: Einsatz des Farbpyrometers am Lepolofen. Zement-Kalk-Gips 17 (1964), 410

6 Z i e g le r, E.: Manteltemperatur und Strahlungsverluste von Drehöfen. Zement-Kalk-Gips 9 (1956), 194.

7 Zieg le r, E.: Manteltemperaturmessungen an Drehöfen. Zement-Kalk-Gips 14 (1961), 179.

8 Lenhart, W. B.: One man, $8 \mathrm{TV}$ cameras control cement making from crusher to raw storage. Rock Products 61 (1958), H. 11, S. $74-77$ und 139.

9 Merkblatt des VDZ, WE 7: Drehofenfeuerungen, 1960.

10 F u n e, G.: Elektroentstauber in der Zementindustrie. Zement-Kalk-Gips 18 (1965), 94.

11 Britisches Patent 1006 444, Inh. Ciments Lafarge, 1961.

12 B e m e $1 \mathrm{~m}$ a $\mathrm{n} \mathrm{n}, \mathrm{W}$.: Rohmaterialmischbetten. Zement-Kalk-Gips 19 (1966), 300.

13 Lahl, W.: Röntgenfluoreszenzanalyse zur Steuerung und Kontrolle im Zementwerk. Zement-Kalk-Gips 18 (1965), 78.

14 Locher, F. W., und Richartz, W.: Schnellbestimmung der chemischen Zusammensetzung von Zementrohstoffen mit physikalischen Verfahren. Tagungsberichte der Zementindustrie, H. 22. I. Teil, 1962, 7. 